\title{
NONLINEAR DIFFERENCE EQUATIONS ANALYTIC IN A PARAMETER
}

\author{
BY \\ C. F. STEPHENS
}

1. Introduction $\left({ }^{1}\right)$. It is probably correct to say that the modern development of the difference calculus began with a memoir by Poincaré published in 1885. Important progress has been made during recent years in the theory of linear equations and there is now a fairly complete theory of linear difference equations. Up to the present time but little progress has been made in the development of a systematic theory of nonlinear difference equations from the point of view of general function theory. However, some progress is being made along these lines.

The main purpose of the present paper is an investigation of the solutions of nonlinear difference equations analytic in a parameter. We are interested in those solutions which are again analytic in this parameter and in the different forms that these solutions may take. In $\$ \$ 2$ and 6 the problems considered in the first two chapters are formulated. The principal results are given in the form of theorems. The method employed is similar to that for differential equations analytic in a parameter. In Chapter III we make certain applications of the results obtained in Chapter II to systems of equations without a parameter. Finally, in Chapter IV we make further investigations of difference equations containing a parameter by applying the results of Chapter III.

\section{Chapter I. Difference equations having no Linear terms IN DEPENDENT VARIABLES AND PARAMETER}

2. Formulation of problem. Consider the system of difference equations having the form

$$
\begin{gathered}
y_{i}(x+1)=f_{i}\left(y_{1}(x), \cdots, y_{n}(x) ; P(x) ; x\right), \\
f_{i}(0, \cdots, 0 ; 0 ; x) \equiv 0 \quad(i=1, \cdots, n),
\end{gathered}
$$

where $P(x)$ represents a parameter that is independent of $y_{i}(x)(i=1, \cdots, n)$ and is periodic of period 1 with respect to $x$.

It is assumed that the $f_{i}$ have the following properties.

Property I. The $f_{i}$ are continuous in $x$ and bounded for all values of the $y_{i}(x), P(x)$ and $x$ for which

Presented to the Society, April 29, 1944 ; received by the editors January 21, 1947, and, in revised form, June 30, 1947.

(1) The author wishes to acknowledge the assistance received from the referee through his valuable criticisms and pertinent comments during the preparation of this paper for publication. 
$G: \quad\left|y_{i}(x)\right| \leqq r_{i} ; \quad|P(x)| \leqq \rho ; \quad|x| \geqq R$.

Property II. The $f_{i}$ are analytic functions of the $y_{i}(x)$ and $P(x)$, but not necessarily analytic functions of $x$, for all values of $y_{i}(x), P(x)$ and $x$ in the domain $G$, and can be developed into convergent power series starting with terms of the second degree in $y_{i}(x)$ and $P(x)$. We make the further assumption that the $f_{i}$ contain terms in $P(x)$ alone. In other words,

$$
\begin{aligned}
& f_{i}=\sum_{m=2}^{\infty} \sum a_{\alpha_{0}, \cdots, \alpha_{n}}^{(i)}(x) P^{\alpha_{0}}(x) y_{1}^{\alpha_{1}}(x) \cdots y_{n}^{\alpha_{n}}(x), \\
& \alpha_{0}, \alpha_{1}, \cdots, \alpha_{n} \geqq 0 ; \alpha_{0}+\alpha_{1}+\cdots+\alpha_{n}=m,
\end{aligned}
$$

where the members on the right converge in the domain $G$ and $a_{2,0, \ldots, 0}^{(i)}(x) \not \equiv 0$.

The assumption that $a_{2,0}^{(i)}, \cdots, 0(x) \not \equiv 0$ is made for simplicity. We need only assume that the $f_{i}$ contain terms in $P(x)$ alone.

3. Formal construction of the solutions of equations (1). We seek a solution having the form

$$
y_{i}(x)=\sum_{j=\sigma}^{\infty} C_{i}^{(j)}(x) P^{j}(x) \quad(i=1, \cdots, n),
$$

where $\sigma=1,2, \cdots, s$ ( $s$ is a finite whole number). Thus,

$$
y_{i}^{\alpha_{i}}(x)=P^{\alpha_{i}}(x)\left[\sum_{j=\sigma}^{\infty} C_{i}^{(j)}(x) P^{j-1}(x)\right]^{\alpha_{i}} \text {. }
$$

Substituting (2a) in the right members of equations (1) we have

$$
\begin{aligned}
y_{i}(x+1)= & \sum_{m=2}^{\infty} P^{m}(x) \sum a_{\alpha_{0}, \cdots, \alpha_{n}}^{(i)}(x) \prod_{\nu=1}^{n}\left[\sum_{j=\sigma}^{\infty} C_{\nu}^{(j)}(x) P^{j-1}(x)\right]^{\alpha_{\nu}} \\
= & \psi_{i}^{(2)}(x) P^{2}(x)+\psi_{i}^{(3)}(x) P^{3}(x)+\cdots \\
& \left(\alpha_{0}, \cdots, \alpha_{n} \geqq 0 ; \alpha_{0}+\cdots+\alpha_{n}=m\right),
\end{aligned}
$$

where $\psi_{i}^{(\nu)}(x)$ are the notations for the coefficients of $P^{\nu}(x)$.

On substituting the $y_{i}(x+1)$ as given by (2) in the left members of equations (3) and on comparing the coefficients of like powers of $P(x)$ in the left and right members of (3), we obtain the following theorem.

THEOREM 1. If the terms in the right members of equations (1) that are of lowest degree in $P(x)$ alone are of degree $\omega$ (a positive whole number), then the system of equations (1) has no solution of the form given by (2) when $\sigma \neq \omega$.

Under our present assumptions $\omega=2$ in (1). By direct calculation of the $\psi_{i}^{\prime}(x)$ by a method due to Trjitzinsky $\left.{ }^{2}\right)$, we have the following theorem.

(2) W. J. Trjitzinsky, Non-linear difference equations, Compositio Math. vol. 5 (19371938) pp. 1-60. 

form

TheOREM 2. The system of equations (1) has a unique formal solution of the

$$
y_{i}(x)=\sum_{k=2}^{\infty} C_{i}^{(k)}(x) P^{k}(x) \quad(i=1, \cdots, n),
$$

where $C_{\mathbf{i}}^{(\mathbf{k})}(x)$ are determined as follows:

$$
C_{i}^{(k)}(x)=\psi_{i}^{(k)}(x-1) \quad(k=2,3, \cdots)
$$

and

$$
\psi_{i}^{(k)}(x)=\left[\sum_{m=2}^{k} \sum^{\prime \prime \prime} a_{\alpha_{0}, \alpha_{1}, \cdots, \alpha_{n}}^{(i)}(x)\right]
$$

$$
\left[\sum^{\prime \prime} \prod_{\nu=1}^{n} \sum^{\prime} C_{\nu}^{\left(j_{1}+1\right)}(x) \cdots C_{\nu}^{\left(j \alpha_{\nu}+1\right)}(x)\right]
$$

where also

(6) $\quad \sum^{\prime}=\sum\left(j_{1}, j_{2}, \cdots, j_{\alpha_{\nu}} \geqq 0 ; j_{1}+j_{2}+\cdots+j_{\alpha_{\nu}}=\beta_{\nu}\right)$,

(7) $\sum^{\prime \prime}=\sum\left(\beta_{1}, \beta_{2}, \cdots, \beta_{n} \geqq 0 ; \beta_{1}+\beta_{2}+\cdots+\beta_{n}=k-m\right)$,

(8) $\quad \sum^{\prime \prime \prime}=\sum\left(\alpha_{0}, \alpha_{1}, \cdots, \alpha_{n} \geqq 0 ; \alpha_{0}+\alpha_{1}+\cdots+\alpha_{n}=m\right)$,

(9) $\quad \sum^{\prime \prime} \prod_{\nu=1}^{n} \sum^{\prime} C_{\nu}^{\left(j_{1}+1\right)}(x) \cdots C_{\nu}^{\left(j \alpha_{\nu}+1\right)}(x) \equiv 1 \quad\left(\alpha_{1}=\alpha_{2}=\cdots=\alpha_{n}=0\right)$.

4. Proof of convergence of the formal solution (2b). We first state a lemma which will enable us to construct a system of difference equations that dominates system (1). Then a particular solution of the system of dominating difference equations is found by solving a system of analytic implicit functions. Finally it is shown that the particular solution of the system of dominating difference equations dominates the solution series (2b) for sufficiently small absolute values of $P(x)$.

LEMMA. If the power series

(10) $f_{i}=\sum_{m=2}^{\infty} \sum^{\prime \prime \prime} a_{\alpha_{0}}^{(i)}, \cdots, \alpha_{n}(x-1) P^{\alpha_{0}}(x) \bar{y}_{1}^{\alpha_{1}}(x) \cdots \bar{y}_{n}^{\alpha_{n}}(x) \quad(i=1, \cdots, n)$ converge absolutely and uniformly for all values of $\bar{y}_{i}(x), P(x)$ and $x$ for which $G_{1}: \quad\left|\bar{y}_{i}(x)\right| \leqq r_{i} ; \quad|P(x)| \leqq \rho ; \quad|x| \geqq R^{\prime}=R+1 \quad(i=1, \cdots, n)$, then there exist other power series

$$
\bar{f}_{i}=\sum_{m=2}^{\infty} \sum^{\prime \prime \prime} A_{\alpha_{0}}^{(i)} \cdots, \alpha_{n} P^{\alpha_{0}}(x) \bar{y}_{1}^{\alpha_{1}}(x) \cdots \bar{y}_{n}^{\alpha_{n}}(x)
$$


which converge absolutely and uniformly for all values of the $\bar{y}_{i}(x)$ and $P(x)$ for which

$G_{2}: \quad\left|\bar{y}_{i}(x)\right| \leqq r_{i}^{\prime}<r_{i} ; \quad|P(x)| \leqq \rho^{\prime}<\rho ; \quad|x| \geqq R^{\prime} ;$

and the coefficients of which are real and positive and satisfy the inequalities

$$
A_{\alpha_{0}, \alpha_{1}, \cdots, \alpha_{n}}^{(i)} \geqq\left|a_{\alpha_{0}, \alpha_{1}, \cdots, \alpha_{n}}(x-1)\right| .
$$

Proof. The proof of the lemma follows from a simple application of the Cauchy integral formula $\left({ }^{3}\right)$.

Consider the system of dominating difference equations

$$
\begin{aligned}
\bar{y}_{i}(x+1) & =\bar{f}_{i}\left(\bar{y}_{1}(x), \cdots, \bar{y}_{n}(x) ; P(x)\right), \\
& \bar{f}_{i}(0, \cdots, 0 ; 0)=0
\end{aligned} \quad(i=1, \cdots, n),
$$

where the $\bar{f}_{i}$ are given by the preceding lemma. Let a formal solution of $\left(1^{\prime}\right)$ be written in the form

$$
\bar{y}_{i}(x)=\bar{C}_{i}^{(2)} P^{2}(x)+\bar{C}_{i}^{(3)} P^{3}(x)+\cdots \quad(i=1, \cdots, n),
$$

where the $\bar{C}_{i}^{(k)}$ are periodic functions of period 1 that are to be so determined that equations $\left(2^{\prime} \mathrm{b}\right)$ shall be a solution of the system of equations $\left(1^{\prime}\right)$. form

Since it follows from $\left(2^{\prime} b\right)$ that $\bar{y}_{i}(x+1)=\bar{y}_{i}(x)$, we can write $\left(1^{\prime}\right)$ in the

$$
\begin{aligned}
\bar{y}_{i}(x)= & \bar{f}_{i}\left(\bar{y}_{1}(x), \cdots, \bar{y}_{n}(x) ; P(x)\right), \\
& \bar{f}_{i}(0, \cdots, 0 ; 0)=0 \quad(i=1, \cdots, n) .
\end{aligned}
$$

It will be shown that the system of equations $\left(1^{\prime} b\right)$ can be solved by the method for solving analytic implicit functions.

If one assumes the convergence of the right members of equations $\left(2^{\prime} \mathrm{b}\right)$ for sufficiently restricted values of $P(x)$, on substituting these series into equations $\left(1^{\prime} \mathrm{b}\right)$, rearranging the right members as power series in $P(x)$, and equating the coefficients of corresponding powers of $P(x)$ in the left and right members, it is found that the conditions that must be satisfied by the $\bar{C}_{i}^{(k)}$ are

$$
\begin{aligned}
& \bar{C}_{i}^{(k)}=\bar{\psi}_{i}^{(k)}=\left[\sum_{m=2}^{k} \sum^{\prime \prime \prime} A_{\alpha_{0}, \cdots, \alpha_{n}}^{(i)}\right] \\
& \cdot\left[\sum^{\prime \prime} \prod_{\nu=1}^{n} \sum^{\prime} \bar{C}_{\nu}^{\left(j_{1}+1\right)} \bar{C}_{\nu}^{\left(j_{2}+1\right)} \cdots \bar{C}_{\nu}^{\left(j_{\alpha_{\nu}}+1\right)}\right],
\end{aligned}
$$

where the summations are to be taken in the same manner as (6), (7), (8) and (9). The $\bar{C}_{i}^{(\boldsymbol{k})}(k=2,3, \cdots ; i=1,2, \cdots, n)$ are uniquely determined by $\left(5^{\prime}\right)$. Hence the formal power series solution $\left(2^{\prime} b\right)$ of equations $\left(1^{\prime} b\right)$ exists and is unique.

(3) F. R. Moulton, Differential equations, 1930, Appendix B. 
Consider the system of analytic implicit functions which dominates the system $\left(1^{\prime} b\right)$

$$
\begin{gathered}
Y_{i}=\bar{M} \frac{P(x)}{\rho^{\prime \prime}}+\frac{\bar{M}}{\left(1-P(x) / \rho^{\prime \prime}\right)\left[1-\left(Y_{1}+\cdots+Y_{n}\right) / r\right]} \\
-\bar{M}\left(1+\frac{Y_{1}+\cdots+Y_{n}}{r}+\frac{P(x)}{\rho^{\prime \prime}}\right), \\
Y_{i}(0, \cdots, 0 ; 0)=0 \quad(i=1, \cdots, n),
\end{gathered}
$$

where $\rho^{\prime \prime}<\rho^{\prime}, r<r_{i}^{\prime}$ and $\bar{M}$ represents an upper bound to the functions $\left|\bar{f}_{i}\left(\bar{y}_{1}(x), \cdots, \bar{y}_{n}(x) ; P(x)\right)\right|$ in the domain $G_{2}$.

It can be shown $\left({ }^{4}\right)$ that the $Y_{i}(=Y)$ converge absolutely and uniformly for all values of $P(x)$ for which the inequality

$$
|P(x)|<\frac{\rho^{\prime \prime}}{1+(4 n / r) \bar{M}[1+(n / r) \bar{M}]}
$$

is satisfied. Thus, it follows that the equations in $\left(2^{\prime} \mathrm{b}\right)$ converge absolutely and uniformly when the inequality (13) is satisfied, and the solution-functions are analytic functions of $P(x)$ under the same condition. This result follows from the form of equations $\left(2^{\prime} b\right)$ and their absolute and uniform convergence. Since the $\bar{f}_{i}$ contain terms in $P(x)$ alone, we do not obtain a solution which reduces identically to zero.

With this we can prove the convergence of the formal power-series solution (2b). It follows from our lemma, (5) and (5') that we must have

$$
\begin{aligned}
\bar{C}_{i}^{(k)}=\bar{\psi}_{i}^{(k)} \geqq\left|\psi_{i}^{(k)}(x-1)\right| & =\left|C_{i}^{(k)}(x)\right| \\
& (k=2,3, \cdots ; i=1, \cdots, n),
\end{aligned}
$$

for

$$
\begin{aligned}
\bar{C}_{i}^{(k)}= & {\left[\sum_{m=2}^{\infty} \sum^{\prime \prime \prime} A_{\alpha_{0}, \alpha_{1}}^{(i)}, \cdots, \alpha_{n}\right] \cdot\left[\sum^{\prime \prime} \prod_{\nu=1}^{n} \sum^{\prime} \bar{C}_{\nu}^{\left(j_{1}+1\right)} \cdots \bar{C}_{\nu}^{\left(j_{\alpha_{\nu}}+1\right)}\right] } \\
\geqq & {\left[\sum_{m=2}^{k} \sum^{\prime \prime \prime}\left|a_{\alpha_{0}}^{(i)}, \cdots, \alpha_{n}(x-1)\right|\right] } \\
& \cdot\left[\sum^{\prime \prime} \prod_{\nu=1}^{n} \sum^{\prime}\left|C_{\nu}^{\left(j_{1}+1\right)}(x-1)\right| \cdots\left|C_{\nu}^{\left(j_{\left.\alpha_{\nu}+1\right)}\right.}(x-1)\right|\right] \\
\geqq & \left|\psi_{i}^{(k)}(x-1)\right|=\left|C_{i}^{(k)}(x)\right| .
\end{aligned}
$$

Since the right members of our solution-functions $\left(2^{\prime} \mathrm{b}\right)$ are absolutely and uniformly convergent for sufficiently small values of $|P(x)|$, it follows that

(`) Ibid. chap. VI. 
the right members of our formal solution-functions (2b) are absolutely and uniformly convergent in a certain domain of the independent variable $x$ when the inequality (13) is satisfied. Therefore, we have proved the following theorem.

THEOREM 3. The system of nonlinear difference equations (1), possessing Properties I and II, has a unique solution of the form (2b) where the functions $C_{i}^{(k)}(x)$ are determined by equations (4) and (5).

5. Solution-series as a function of $x$ and $P(x)$. One can easily find periodic functions of period 1 which satisfy the inequality (13). Therefore, it follows from the form and convergence of equations (2b) that the solution-functions are analytic in the parameter $P(x)$ for all values of $P(x)$ and $x$ for which equations $(2 b)$ converge.

Let $C$ be a circle with center at the origin and radius $R^{\prime}$. Draw tangents $l$ and $l^{\prime}$ to the circle $C$ at the points where the circle cuts the axis of imaginaries. Denote by $D$ all points not interior to the domain bounded by that part of circle $C$ which lies to the left of the axis of imaginaries and the parts of tangents $l$ and $l^{\prime}$ which do not lie to the left of this axis. Thus, the domain $D$ contains no part of the positive axis of reals. It follows from Property $I$, equations (4) and (5), that the functions $C_{t}^{(\nu)}(x)$ of equations (2b) are continuous in $D$. We shall now assume that $P(x)$ is continuous in $D$.

Let us write $(2 b)$ in the form

$$
y_{i}(x)=\sum_{\nu=2}^{\infty} g_{i}^{(\nu)}(x) \quad(i=1, \cdots, n),
$$

where $g_{i}^{(\nu)}(x) \equiv C_{i}^{(\nu)}(x) P^{\nu}(x)$. Since the $g_{i}^{(\nu)}(x)$ are continuous functions of $x$ and the right members of equations (2b) are absolutely and uniformly convergent in the domain $D$ when the inequality (13) is satisfied, it follows that the $y_{i}(x)$ are continuous functions of $x$. We can now state the following theorem.

THEOREM 4. If $x$ is in the domain $D$ and $P(x)$ satisfies the inequality (13), the solution-functions (2b) are continuous functions of $x$ and analytic functions of $P(x)$.

Chapter 2. Difference equations having a parameter as A FACTOR OF THEIR RIGHT MEMBERS

6. Formulation of problem. Let us now consider the system of nonlinear difference equations having the form

$$
\begin{aligned}
& y_{i}(x+1)=P(x) f_{i}\left(y_{1}(x), \cdots, y_{n}(x) ; P(x) ; x\right), \\
& f_{i}(0, \cdots, 0 ; 0 ; x)=a_{i}(x) \not \equiv 0 \quad(i=1, \cdots, n),
\end{aligned}
$$


where $P(x)$ has the same meaning as before. The functions $a_{i}(x)$ are taken to be independent of $P(x)$. It is assumed that the $f_{i}$ are analytic functions of the $y_{i}(x)$ and $P(x)$, and continuous functions of $x$ in the domain $G$. In other words, the system of equations (15) can be written in the form

$$
\begin{array}{r}
y_{i}(x+1)=a_{i}(x) P(x)+\sum_{m=2}^{\infty} \sum a_{\alpha_{0}}^{(i)} \cdots, \alpha_{n}(x) P^{\alpha_{0}}(x) y_{1}^{\alpha_{1}}(x) \cdots y_{n}^{\alpha_{n}}(x), \\
\alpha_{0} \geqq 1 ; \alpha_{1}, \cdots, \alpha_{n} \geqq 0 ; \alpha_{0}+\cdots+\alpha_{n}=m,
\end{array}
$$

where the right members converge and are bounded in the domain $G$.

Let us seek all solutions of equations (15) having the form

$$
y_{i}(x)=\sum_{j=\sigma}^{\infty} C_{i}^{(j)}(x) P^{j}(x) \quad(i=1, \cdots, n),
$$

where $\sigma=0,1, \cdots, s$ ( $s$ being a finite positive whole number).

7. Formal construction of the solution. The proof of the existence of a unique solution of equations (15) is by the same general method as that employed in Chap. I. There exists a formal solution of equations (15) in the power-series form

$$
y_{i}(x)=\sum_{j=1}^{\infty} C_{i}^{(j)}(x) P^{j}(x) \quad(i=1, \cdots, n),
$$

where the $C_{t}^{(j)}(x)$ are functions of $x$ which are to be determined.

On substituting equations (17) into equations (15), rearranging the terms according to powers of $P(x)$, it is found that the $C_{i}^{(j)}(x)$ must satisfy

$$
\begin{aligned}
C_{i}^{(1)}(x+1) & =f_{i}(0, \cdots, 0 ; 0 ; x)=a_{i}(x), \\
C_{i}^{(2)}(x+1) & =\sum_{j=1}^{n} \frac{\partial f_{i}}{\partial y_{j}} C_{j}^{(1)}(x)+\frac{\partial f_{i}}{\partial P}, \\
C_{i}^{(k)}(x+1) & =\psi_{i}^{(k)}\left(C_{j}^{(1)}(x), \cdots, C_{j}^{(k-1)}(x) ; x\right) \\
& (k=2,3, \cdots ; j=1, \cdots, n),
\end{aligned}
$$

where the $\psi_{i}^{(k)}$ are polynomials in the $C_{j}^{(1)}(x), \cdots, C_{j}^{(k-1)}(x)$, but not in $x$, whose coefficients are linear functions of the coefficients of the expansions of the functions $f_{i}$ as power series in the $y_{i}(x)$ and $P(x)$, and the coefficients have positive numerical multipliers. The $y_{i}(x)$ and $P(x)$ are replaced by zero in all partial derivatives. The $C_{i}^{(\boldsymbol{k})}(x)$ are uniquely determined by equations (18).

8. Proof of convergence of formal solution (17). Let us consider the system of difference equations having the form 


$$
\begin{gathered}
\bar{y}_{i}(x+1)=A_{i} P(x)+\sum_{m=2}^{\infty} \sum A_{\alpha_{0}, \cdots, \alpha_{n}}^{(i)} P^{\alpha_{0}}(x) \bar{y}_{1}^{\alpha_{1}}(x) \cdots \bar{y}_{n}^{\alpha_{n}}(x) \\
=P(x) \bar{f}_{i}\left(\bar{y}_{2}(x), \cdots, \bar{y}_{n}(x) ; P(x)\right), \\
\bar{f}_{i}(0, \cdots, 0 ; 0)=A_{i} \quad(i=1, \cdots, n), \\
\alpha_{0} \geqq 1 ; \alpha_{1}, \cdots, \alpha_{n} \geqq 0 ; \alpha_{0}+\cdots+\alpha_{n}=m .
\end{gathered}
$$

where $A_{i} \geqq\left|a_{i}(x-1)\right| ; a_{\alpha_{0}, \alpha_{1}, \cdots, \alpha_{n}}^{(i)} \geqq\left|a_{\alpha_{0}, \alpha_{1}, \cdots \alpha_{n}}^{(i)}(x-1)\right|$. The right members of equations $\left(15^{\prime}\right)$ converge for all values of the $\bar{y}_{i}(x)$ and $P(x)$ in the domain $G_{2}$.

The existence of the system of equations $\left(15^{\prime}\right)$ in the domain $G_{2}$ is established by a discussion similar to that in proving the lemma of $\S 4$.

A formal solution of $\left(15^{\prime}\right)$ may be written in the form

$$
\bar{y}_{i}(x)=\sum_{k=1}^{\infty} \bar{C}_{i}^{(k)} P^{k}(x) \quad(i=1, \cdots, n),
$$

where the $\bar{C}_{i}^{(k)}$ are constants. It is found that the $\bar{C}_{i}^{(k)}$ must satisfy the equations

$$
\begin{aligned}
\bar{C}_{i}^{(1)} & =A_{i}, \\
\bar{C}_{i}^{(2)} & =\sum_{j=1}^{n} \frac{\partial \bar{f}_{i}}{\partial \bar{y}_{j}} \bar{C}_{j}^{(1)}+\frac{\partial \bar{f}_{i}}{\partial P}, \\
\bar{C}_{i}^{(k)} & =\bar{\psi}_{i}^{(k)}\left(\bar{C}_{j}^{(1)}, \cdots, \bar{C}_{j}^{(k-1)}\right) \quad(k=2,3, \cdots),
\end{aligned}
$$

where the $\bar{\psi}_{i}^{(k)}$ have the same general properties as the $\psi_{i}^{(k)}$.

Since it follows from $\left(17^{\prime}\right)$ that $\bar{y}_{i}(x+1)=\bar{y}_{i}(x)$, we can write $\left(15^{\prime}\right)$ in the form

$$
\bar{y}_{i}(x)=P(x) \bar{f}_{i}\left(\bar{y}_{1}(x), \cdots, \bar{y}_{n}(x) ; P(x)\right) \quad(i=1, \cdots, n) .
$$

The system of equations (15'a) will be solved by the method for solving analytic implicit functions.

Consider the system of analytic implicit functions

$$
Y_{i}=\frac{P(x) \bar{M}}{\left(1-P(x) / \rho^{\prime \prime}\right)\left[1-\left(Y_{1}+\cdots+Y_{n}\right) / r^{\prime \prime}\right]} \quad(i=1, \cdots, n) .
$$

If $r^{\prime \prime}<r_{i}^{\prime}, \rho^{\prime \prime}<\rho^{\prime}$ and $\bar{M} \geqq\left|\bar{f}_{i}\left(\bar{y}_{1}(x), \cdots, \bar{y}_{n}(x) ; P(x)\right)\right|$ for all values of the $\bar{y}_{i}(x)$ and $P(x)$ in the domain $G_{2}$, the expansions of the right members of equations (19) would dominate the expansions of the right members of equations (15'a). The constants $r^{\prime \prime}, \rho^{\prime \prime}$ and $\bar{M}$ will be so chosen that such is the case.

It follows from equations (19) that $Y_{1}=\cdots=Y_{n}=Y$ and that $Y$ satisfies 
the equation

$$
Y=\frac{P(x) \bar{M}}{\left(1-P(x) / \rho^{\prime \prime}\right)\left(1-n Y / r^{\prime \prime}\right)} .
$$

The solution of this equation for $Y$, vanishing with $P(x)=0$, is

$$
2 \frac{n}{r^{\prime \prime}} Y=1-\left(1-\frac{4 n}{r^{\prime \prime}} \bar{M} \frac{P(x)}{1-P(x) / \rho^{\prime \prime}}\right)^{1 / 2} \text {. }
$$

It is found that the expansions of the right members of (19) as power series in $P(x)$ converge absolutely and uniformly for all values of $P(x)$ such that

$$
|P(x)|<\frac{\rho^{\prime \prime}}{1+4 n \bar{M} \rho^{\prime \prime} / r^{\prime \prime}},
$$

since the singular point of the expansion of the right member of equation (20) as a power series in $P(x)$ is defined by the equation

$$
r^{\prime \prime}\left(1-\frac{P(x)}{\rho^{\prime \prime}}\right)=4 n \bar{M} P(x) .
$$

It follows that the right members of the formal solution $\left(17^{\prime}\right)$ of equations $\left(15^{\prime}\right)$ converge absolutely and uniformly under the condition that the inequality (21) is satisfied.

We can now prove the convergence of the formal solution (17). On making use of the fact that the expansions of the functions $\bar{f}_{i}\left(\bar{y}_{1}(x), \cdots, \bar{y}_{n}(x) ; P(x)\right)$ dominate the expansions of the functions $f_{i}\left(y_{1}(x), \cdots, y_{n}(x) ; P(x) ; x\right)$, and on comparing the first set of equations (18) with the first set of equations $\left(18^{\prime}\right)$, it is found that $\bar{C}_{i}^{(1)}=A_{i} \geqq\left|a_{i}(x-1)\right|=\left|C_{i}^{(1)}(x)\right|(i=1, \cdots, n)$. Then it follows from the second set of equations (18) and (18') that

$$
\bar{C}_{i}^{(2)} \geqq\left|C_{i}^{(2)}(x)\right| ;
$$

and it follows sequentially from these results and the properties of the $\bar{\psi}_{i}^{(k)}$ and the $\psi_{i}^{(k)}$ that

$$
\bar{C}_{i}^{(k)} \geqq\left|C_{i}^{(k)}(x)\right| \quad(i=1, \cdots, n ; k=1,2, \cdots) .
$$

Therefore the power series $\left(17^{\prime}\right)$ dominate the power series (17). Since the power series $\left(17^{\prime}\right)$ converge absolutely and uniformly when the inequality (21) is satisfied, the solution-series (17) also converge absolutely and uniformly under the same condition when $x$ is in the domain $D$ and therefore form an actual solution of equations (15).

The solution-series (17) are analytic functions of $P(x)$ and continuous functions of $x$. It can be shown by direct substitution that the system of equations (15) has no other solution of the form (16). Therefore, we can now state the following theorem. 
THEOREM 5. The system of nonlinear difference equations (15) has a unique solution having the form

$$
y_{i}(x)=\sum_{k=1}^{\infty} C_{i}^{(k)}(x) P^{k}(x) \quad(i=1, \cdots, n),
$$

where the $C_{i}^{(k)}(x)$ are given by equations (18), and the solution-functions are analytic functions of $P(x)$ whenever the inequality (21) is satisfied and continuous functions of $x$ in the domain $D(\$ 4)$ of the independent variable $x$.

Chapter III. Applications to systems of equations WITHOUT A PARAMETER

9. A special theorem. We shall now make use of the results obtained in the last chapter. Let us consider the system of nonlinear difference equations

$$
\begin{gathered}
y_{i}(x+1)=f_{i}\left(y_{1}(x), \cdots, y_{n}(x) ; x\right), \\
f_{i}(0, \cdots, 0 ; x)=a_{i}(x) \not \equiv 0 \quad(i=1, \cdots, n),
\end{gathered}
$$

where the functions $f_{i}$ are analytic functions of the $y_{i}(x)$, but not necessarily analytic functions of $x$, and can be developed into convergent power series starting with terms independent of $y_{i}(x)$. We shall also assume that the $f_{i}$ satisfy the following condition.

Condition A. We can determine positive constants $R$ and $r$ such that

$$
\left|f_{i}\left(y_{1}(x), \cdots, y_{n}(x) ; x\right)\right|<r / 4 n
$$

for $\left|y_{i}(x)\right| \leqq r ;|x| \geqq R$. We say therefore that $R$ and $r$ satisfy Condition A.

We shall prove the following theorem.

THEOREM 6. If $x$ is in the domain $D$, where $R^{\prime}=R+1$, and if $R$ and $r$ satisfy Condition A, then the system of equations (22) has a solution whose solutionfunctions are continuous in $x$.

Proof. Take for consideration the system of equations

$$
y_{i}(x+1)=P f_{i}\left(y_{1}(x), \cdots, y_{n}(x) ; x\right) \quad(i=1, \cdots, n),
$$

where $P$ is a positive constant which later shall be put equal to 1 . Equations (15) include equations $\left(22^{\prime}\right)$ as a special case. From Theorem 5 it follows that the system of equations $\left(22^{\prime}\right)$ has a unique continuous solution having the form

$$
y_{i}(x)=\sum_{k=1}^{\infty} C_{i}^{(k)}(x) P^{k} \quad(i=1, \cdots, n),
$$

where the $C_{i}^{(k)}(x)$ must satisfy the equations 


$$
\begin{aligned}
C_{i}^{(1)}(x+1) & =f_{i}(0, \cdots, 0 ; x)=a_{i}(x), \\
C_{i}^{(2)}(x+1) & =\sum_{j=1}^{n} \frac{\partial f_{i}}{\partial y_{j}} C_{j}^{(1)}(x), \\
C_{i}^{(k)}(x+1) & =\psi_{i}^{(k)}\left(C_{j}^{(1)}(x), \cdots, C_{j}^{(k-1)}(x) ; x\right) \\
& (k=2,3, \cdots ; i, j=1, \cdots, n) ;
\end{aligned}
$$

here the $\psi_{i}^{(k)}$ are polynomials in the $C_{j}^{(1)}(x), \cdots, C_{j}^{(k-1)}(x)$, but not in $x$, whose coefficients are linear functions of the coefficients of the expansions of the functions $f_{i}$ as power series in the $y_{i}(x)$, and have positive numerical multipliers. The $y_{i}(x)$ are replaced by zero in all partial derivatives.

Equations (23) converge absolutely and uniformly for all values of $P$ such that (21) is satisfied when $x$ is in the domain $D$. Since the functions $f_{i}$ do not depend upon $P$, the constants $\rho$ in $G, \rho^{\prime}$ in $G_{2}$, and $\rho^{\prime \prime}$ in (19) are infinite. The limit of the inequality (21) for $\rho^{\prime \prime}=\infty$ is

$$
P<r^{\prime \prime} / 4 n \bar{M} \text {. }
$$

In the problem under consideration $P=1$, and the inequality (25) gives

$$
\bar{M}<r^{\prime \prime} / 4 n
$$

as a sufficient condition for the absolute and uniform convergence of

$$
y_{i}(x)=\sum_{k=1}^{\infty} C_{i}^{(k)}(x) \quad(i=1, \cdots, n) .
$$

From Condition A we obtain $\left|f_{i}\left(y_{1}(x), \cdots, y_{n}(x) ; x\right)\right| \leqq \bar{M}<r / 4 n$. Since $r^{\prime \prime}$ differs from $r$ by a constant as small as one wishes, the inequality (26) is satisfied. This completes the proof of Theorem 6 .

10. Applications. Let us first consider the system of equations

$$
x^{k} y_{i}(x+1)=g_{i}\left(y_{1}(x), \cdots, y_{n}(x) ; x\right) \quad(i=1, \cdots, n),
$$

where the functions $g_{i}$ are analytic functions of the $y_{i}(x)$, but not necessarily analytic functions of $x$, and can be developed into power series starting with terms independent of the $y_{i}(x)$ and these power series are convergent in the domain

$G_{3}$ :

$$
|y \cdot(x)| \leqq r_{i} ; \quad|x| \geqq K .
$$

The number $k$ is any positive constant.

It is obvious that equations (27) can be put in the form of equations (22) and that Condition $\mathrm{A}$ is satisfied. Therefore, it follows from Theorem 6 that the system of equations (27) has a solution which is continuous in $x$ in the domain $D$. Also, these solution-functions approach zero as $x$ approaches infinity in this domain. 
For example, the simple nonhomogeneous linear difference equation

$$
x y(x+1)=b+a y(x),
$$

where $a$ and $b$ are positive constants, is a special case of equations (27). This equation has the particular continuous solution

$$
y(x)=\frac{b}{x-1}+\frac{a b}{(x-1)(x-2)}+\cdots+\frac{a^{k-1} b}{(x-1) \cdots(x-k)}+\cdots,
$$

which is valid for $x$ in a domain defined by Condition $\mathrm{A}$ and has poles at points congruent on the right to the point $x=0$.

Now consider the system of equations

$$
y_{i}(x+1)=h_{i}\left(y_{1}(x), \cdots, y_{n}(x) ; x\right) \quad(i=1, \cdots, n),
$$

where

$$
h_{i}=x^{-2} a_{i}(x)+x^{-1} \sum_{j=1}^{n} b_{i j}(x) y_{j}(x)+l_{i}\left(y_{1}(x), \cdots, y_{n}(x) ; x\right)
$$

The functions $l_{i}$ can be developed into convergent power series in the $y_{i}(x)$, but not necessarily in $x$, starting with terms of the second degree in these variables and vanishing with them. These developments are valid in the domain $G_{3}$. The functions $b_{i j}(x)$ and $a_{i}(x)$ are any continuous functions of $x$ which are bounded in $G_{3}$ such that the above developments are valid, provided the $a_{i}(x)$ are not identically equal to zero.

The transformation

$$
y_{i}(x)=x^{-1} z_{i}(x) \quad(i=1, \cdots, n)
$$

will transform equations (28) into a system of the form (22) such that Condition A is satisfied. Therefore, it follows from Theorem 6 and (29) that the system of equations (28) has a continuous solution in a certain domain of the independent variable $x$.

Finally, consider the system of equations

$$
y_{i}(x+1)=q_{i}\left(y_{1}(x), \cdots, y_{n}(x) ; x\right) \quad(i=1, \cdots, n),
$$

where

$$
\begin{aligned}
q_{i}-x^{k} a_{i}(x)= & x^{-1} \sum_{j=1}^{n} b_{i j}(x) y_{j}(x) \\
& +\sum_{m=2}^{\infty} \sum x^{-m(k+1)} a_{\alpha_{1}}, \cdots, \alpha_{n}(x) y_{1}^{\alpha_{1}}(x) \cdots y_{n}^{\alpha_{n}}(x), \\
& \quad k \geqq 0 ; \alpha_{1}, \cdots, \alpha_{n} \geqq 0 ; \alpha_{1}+\cdots+\alpha_{n}=m .
\end{aligned}
$$


The functions $a_{i}(x), b_{i j}(x)$, and $a_{\alpha_{1}}, \ldots, \alpha_{n}(x)$ are any functions of $x$ which are continuous and bounded in $G_{3}$ such that the right members of (30a) converge.

The transformation

$$
y_{i}(x)=x^{k+1} z_{i}(x) \quad(i=1, \cdots, n)
$$

will transform equations (30) into a system of the form (27). Thus it follows that there exists a continuous solution of equations (30).

\section{Chapter IV. Further Developments Concerning Difference EQUATIONS WITH A PARAMETER}

11. Nonlinear equations. In Chapters I and II we assumed that the functions $f_{i}$, as defined by equations (1) and (15), were analytic in the parameter $P(x)$ and contained no linear terms in the dependent variables alone. However, we required the functions $f_{i}$ to be continuous and bounded in $x$ in a certain domain, but not necessarily analytic in $x$. In view of the developments in Chapter III we can find solutions of difference equations with more general assumptions concerning the $f_{i}$ as functions of the parameter $P(x)$, provided we make more restrictive assumptions concerning the $f_{i}$ as functions of $x$.

For simplicity we shall write $p$ for $P(x)$, where $P(x)$ is considered to be a periodic function of $x$ of period 1. Take for consideration the system of equations

$$
x^{k} y_{i}(x+1)=f_{i}\left(y_{1}(x), \cdots, y_{n}(x) ; p ; x\right),
$$

where the $f_{i}$ are analytic in the $y_{i}(x)$, continuous in $p$ and $x$, and can be developed into convergent power series in the $y_{i}(x)$ in the domain $G$. We take $k$ to be a positive number. In other words, we may write

$$
\begin{aligned}
& f_{i}=a_{i}(x, p)+\sum_{m=1}^{\infty} \sum a_{\alpha_{1}}^{(i)} \cdots, \alpha_{n}(x, p) y_{1}^{\alpha_{1}}(x) \cdots y_{n}^{\alpha_{n}}(x), \\
& \cdot \alpha_{1}, \cdots, \alpha_{n} \geqq 0 ; \alpha_{1}+\cdots+\alpha_{n}=m,
\end{aligned}
$$

where the right members converge in $G$ and $a_{i}(x, p) \not \equiv 0$.

It follows from a discussion similar to that given for equations (27) that the system of equations $\left(27^{\prime}\right)$ has a solution which is continuous in $x$ and $p$.

Likewise, from discussions similar to those given for equations (28) and (30) it follows that each of the following systems of equations has a solution which is continuous in $x$ and $p$ :

$$
\begin{aligned}
y_{i}(x+1)=x^{-2} a_{i}(x, p) & +x^{-1} \sum_{j=1}^{n} b_{i j}(x, p) y_{j}(x) \\
& +\sum_{m=2}^{\infty} \sum a_{\alpha_{1}}, \ldots, \alpha_{n}(x, p) y_{1}^{\alpha_{1}}(x) \cdots y_{n}^{\alpha_{n}}(x)
\end{aligned}
$$




$$
\begin{aligned}
& y_{i}(x+1)-x^{k} a_{i}(x, p)=x^{-1} \sum_{j=1}^{n} b_{i j}(x, p) y_{j}(x) \\
&+\sum_{m=2}^{\infty} \sum x^{-m(k+1)} a_{\alpha_{1}}, \cdots, \alpha_{n}(x, p) y_{1}^{\alpha_{1}}(x) \cdots y_{n}^{\alpha_{n}}(x), \\
& k \geqq 0 ; \alpha_{1}, \cdots, \alpha_{n} \geqq 0 ; \alpha_{1}+\cdots+\alpha_{n}=m ;
\end{aligned}
$$

where the right members of $\left(28^{\prime}\right)$ and $\left(30^{\prime}\right)$ converge in $G$ and where the functions $a_{i}(x, p), b_{i j}(x, p)$ and $a_{\alpha_{1}}, \ldots, \alpha_{n}(x, p)$ are continuous and bounded in $x$ and $p$, but not necessarily analytic in the independent variable $x$ and parameter $p$.

12. Linear equations. Consider the system of linear difference equations

$$
y_{i}(x+1)=x^{k} a_{i}(x, p)+x^{-1} \sum_{j=1}^{n} b_{i j}(x, p) y_{j}(x) \quad(i=1, \cdots, n),
$$

where the $a_{i}(x, p)$ and the $b_{i j}(x, p)$ are any functions of $x$ and $p$ which are continuous and bounded in $x$ and $p$ in the domain

$G_{4}$ :

$$
|p| \leqq \rho ; \quad|x| \geqq K .
$$

If $k$ is a negative number, the system of equations (32) becomes a special case of system $\left(27^{\prime}\right)$. If $k$ is zero or a positive number, the system of equations (32) becomes a special case of system $\left(30^{\prime}\right)$. In each of these cases we know that the system of equations (32) has a solution which is continuous in $x$ and $p$.

Finally, consider the system of equations

$$
y_{i}(x+1)=a_{i}(x, p)+\sum_{j=1}^{n} b_{i j}(x, p) y_{j}(x) \quad(i=1, \cdots, n),
$$

where the functions $a_{i}(x, p)$ and $b_{i j}(x, p)$ are analytic in the parameter $p$ and continuous in $x$ in the domain $G_{4}$, and can be developed into convergent power series in $p$, but not necessarily in $x$. We shall also assume that $a_{i}(x, 0)$ and $b_{i j}(x, 0)$ are identically equal to zero.

It can easily be shown that the system of equations (33) is a special case of system (15). Therefore, it follows from Theorem 5 that the system of equations (33) has a unique solution which is continuous in $x$ and analytic in $p$.

\section{BIBLIOGRAPHY}

R. D. Carmichael, The present state of the difference calculus and the prospect for the future, Amer. Math. Monthly vol. 31 (1924) pp. 169-183.

Heinrich Löwig, Zur Theorie der nichtlinearen Differenzengleichungen, Acta Math. vols. 57-58, pp. 293-340. 
J. Horn, Zur Theorie nichtlinearen Differential und Differenzengleichungen, J. Math. Pures Appl. vol. 141 (1912) pp. 200-216.

, Über eine nichtlineare Differenzengleichung, Jber. Deutschen Math. Verein. vol. 26, pp. 230-251.

F. R. Moulton, Differential equations, New York, Macmillan, 1930.

N. E. Nörlund, Neuere Untersuchungen über Differenzengleichungen, Encyklopädie der Mathematischen Wissenschaften II, 3-2, 1923, pp. 705-711.

Oskar Perron, Über Stabilität und asymptotisches Verhalten der Lösungen eines System endlicher Differenzengleichungen, Journal für Mathematik vol. 161, pp. 41-64. 141.

Ta Li, Die Stabilitätsfrage bei Differenzengleichungen, Acta Math. vol. 63 (1934) pp. 99-

W. J. Trjitzinsky, Non-linear difference equations, Compositio Math. vol. 5 (1937-1938) pp. $1-60$.

, Concerning linear difference equations contcining a parameter, Annali di Matematica (4) vol. 14 (1936) pp. 181-214.

UNIVERSITY OF MICHIGAN, ANN ARbor, Mich.

Prairie View University, Prairie View, Tex. 